\title{
Geometria fractal no ensino médio: teoria e prática
}

\author{
Ivana Côrtes Gladson Antunes
}

\begin{abstract}
Resumo
Neste artigo apresenta-se uma proposta de atividade tendo como objetivo utilizar a Geometria Fractal no Ensino Médio regular, onde se pode trabalhar alguns conceitos matemáticos em suas diversas áreas, tais como álgebra, cálculo, geometria plana e espacial, progressões e logaritmo de maneira diferente da convencional. Dessa forma, pretende-se reforçar a ideia atual da necessidade de experimentar a Matemática por caminhos diferentes para além da resolução de exercícios repetitivos e sem nenhum sentido lógico para o aluno. Neste sentido, a Geometria Fractal permite explorar diversos conceitos matemáticos de uma maneira mais dinâmica e criativa, por meio da construção de modelos e tabelas com os resultados das iterações, chegando a uma dedução geral do que está ocorrendo. Essa proposta é derivada de um Trabalho de Conclusão de Curso do Programa de Pós-graduação em Matemática PROFMAT, polo UNIRIO.
\end{abstract}

Palavras-chave: Geometria Fractal; fractal; inovação.

\section{Abstract}

In this article we present a proposal for an activity with the objective of using Fractal Geometry in regular secondary education, where we can work some mathematical concepts in its various areas, such as algebra, calculus, flat and spatial geometry, progressions and logarithm in a different way of the conventional. In this way, we intend to reinforce the current idea of the need to experiment with mathematics in different ways beyond the resolution of repetitive exercises and without any logical sense for the student. In this sense, Fractal Geometry allows us to explore several mathematical concepts in a more dynamic and creative way, through the construction of models and tables with the results of the iterations, arriving at a general deduction of what is taking place. This proposal is derived from a dissertation of the PROFMAT Mathematics Graduate Program, UNIRIO.

Keywords: Fractal Geometry; fractal; innovation.

\section{Introdução}

No início de dezembro de 2013, foi divulgado ${ }^{1}$ mais um ranking da avaliação internacional do PISA (Programa Internacional de Avaliação dos Estudantes) e ficamos na $58^{\underline{a}}$ posição, em Matemática,

\footnotetext{
${ }^{1}$ Fonte: <http://g1.globo.com/educacao/noticia/2013/12> em 23 de dezembro de 2013.
} 
entre as 65 nações que participam da avaliação, obtendo um total de 391 pontos. Apresentamos uma "tímida" evolução, pois em 2009 conseguimos 386 pontos. Esta prova é realizada por estudantes de 15 anos de idade matriculados na rede pública ou privada de ensino a partir do $7^{\circ}$ ano do Ensino Fundamental.

Este resultado vem ao encontro com a nossa opinião de que no Brasil, principalmente na educação básica, boa parte dos alunos apresentam grandes dificuldades em Matemática, muito em consequência do desinteresse pelo ensino da disciplina e a não associação do que aprende em sala de aula com a aplicação no cotidiano. Sabemos que, hoje, a grande maioria dos livros didáticos e dos professores tentam trabalhar com alguma contextualização, mas ainda não é suficiente para gerar um grande interesse nos alunos, não somente pelo estudo da Matemática, mas pelo conhecimento de forma geral.

Este trabalho surgiu a partir da intenção de propor aulas onde o aluno possa descobrir e fazer relações entre o que visualiza e o que estuda, tentando tornar o conteúdo trabalhado em sala de aula favorável a aprendizagem significativa do aluno. Segundo [5]: ,

A exploração da geometria fractal, em contexto de sala de aula, proporciona o desenvolvimento das atitudes, dos valores e das competências dos alunos, na medida em que promove a curiosidade e o gosto de aprender, de pesquisar e de investigar; impulsiona a utilização da matemática na interpretação do real, reconhecendo formas $e$ processos que envolvem conceitos matemáticos; ajuda na compreensão dos conceitos de perímetro, área e volume; promove a pesquisa de padrões e regularidades formulando em seguida generalizações em situações diversas, nomeadamente em contextos numéricos e geométricos. Propor uma aula com situações novas, onde o educando possa descobrir e fazer relações entre o que visualiza e o que estuda, torna o acontecimento em sala de aula favorável a aprendizagem. Esta abordagem possibilitará ao educando a visualização do conteúdo trabalhado, não ficando apenas na formalidade que é própria da disciplina de matemática. Além do campo extenso de aplicações dos fractais é necessário que o professor perceba a potencialidade que existe nesta área da geometria, podendo assim trabalhar conceitos de simetria, relacionando arte com matemática. [5]

Será apresentado um breve resumo sobre o surgimento e as aplicações nas ciências e tecnologias da Geometria Fractal, mostrando que existem diversas áreas que utilizam esta geometria para a solução de seus problemas, tentando dessa forma despertar não só no aluno, mas também no nosso colega professor, o interesse em aplicar esses conhecimentos na sua sala de aula, tornando as mesmas mais dinâmicas e atraentes.

As atividades que serão apresentadas foram aplicadas para alunos da Escola SESC de Ensino Médio $^{2}$ durante a realização do Projeto de Iniciação Científica da instituição, que tem por objetivo discutir e produzir saberes a partir de um trabalho de pesquisa no qual orientadores de todas as áreas conduzem grupos de alunos no processo de investigação.

Serão analisados o comportamento de dois fractais clássicos: a ilha de Von Koch que tem

\footnotetext{
${ }^{2}$ Localizada no Rio de Janeiro, é uma escola-residência, inteiramente gratuita, que atende a alunos de todo o país, com idades entre 13 e 18 anos. Inaugurada em 2008, hoje possui aproximadamente 500 estudantes nas três séries do Ensino Médio, que moram nas vilas residenciais, junto com professores e gestores. Mais informações,
} $<$ http://escolasesc.edt.com.br>. 
perímetro infinito e área finita e o triângulo de Sierpinski que possui perímetro infinito e área zero. Ao final do artigo é apresentado um breve tutorial do software gratuito "Xaos" versão 3.5, esse programa pode ser utilizado para realizar com os alunos algumas atividades animadas e interativas com fractais.

\title{
2. Fractais: origem e aplicações
}

A Geometria Euclidiana é a geometria que normalmente aprendemos nas escolas. Seus polígonos e poliedros regulares fazem parte da história da Matemática, pois serviram de base para a compreensão da Natureza através da ciência. As criações humanas servem-se majoritariamente das formas geométricas euclidianas para as suas construções, como por exemplo, edifícios, objetos industriais e do cotidiano. Em Batanete encontramos: ,

\begin{abstract}
Por tradição conta-se que há mais de dois mil anos, Euclides enquanto caminhava pela praia, notou que a areia, vista como um todo, se assemelhava a uma superfície contínua e uniforme, embora fosse composta por pequenas partes visíveis. Desde então Euclides tentou provar, matematicamente, que todas as formas da natureza podiam ser reduzidas as formas geométricas simples. Em alguns casos, continua a fazer sentido sua utilização, como por exemplo, o uso da esfera como aproximação do modelo da forma da Terra, da elipse como modelo das órbitas celestes e da parábola como trajetória dos projéteis. [2]
\end{abstract}

Euclides concentrou-se sobretudo nas formas, deixando de lado, um elemento importantíssimo neste tipo de análise, a dimensão. Existe uma infinidade de fenômenos na natureza que não podem ser descritos por essa geometria. A maior parte das formas apresentadas pela Natureza, não são regulares e nem suaves, pelo contrário, são extremamente complexas, recortadas e irregulares. É o caso de grande parte das árvores e plantas, das rochas e das nuvens.

Como disse Mandelbrot: ,

Nuvens não são esferas, montanhas não são cones, continentes não são círculos, tronco de árvores não são suaves e nem o relâmpago viaja em linha reta. [4]

Na segunda metade do século XIX e na primeira metade do século XX, alguns matemáticos descreveram objetos que ficaram conhecidos como "monstros matemáticos", como por exemplo: a curva de Peano, triângulo de Sierpinski, a curva de Von Koch, o conjunto de Julia, o conjunto de Cantor, entre outros.

Benoit Mandelbrot, um matemático que possuia uma visão geométrica aguçada, não via com bons olhos a crescente algebrização da Matemática praticada por Bourbaki, na primeira metade do século XX, na França. Esse foi um dos motivos que levou Mandelbrot a se mudar para o Estados Unidos, em 1948, para trabalhar no Instituto de Pesquisa James Watson da IBM.

Mandelbrot trabalhou em várias pesquisas e uma delas começou pela indagação: "Quanto mede o litoral da Grã-Bretanha?" A resposta encontrada por Mandelbrot, segundo Barbosa, foi: ,

A resposta possível variará conforme a escala de medição. Baías e penínsulas aparecerão ou não, dependendo da escala adotada. Sabe-se, por exemplo, que em um 
documento dos dois países vizinhos, a fronteira de Espanha e Portugal difere em cerca de 20\%, o mesmo acontecendo por exemplo com a fronteira da Holanda e da Bélgica. Claro que ao efetuar as medidas cada país empregou instrumentos com unidade de escalas diferentes. [1]

Com isso, ele quis dizer que para medir o tamanho de um litoral ou limite territorial, é preciso, antes de tudo, definir a escala. Matematicamente falando a escala para medir com exatidão uma extensão territorial deveria tender a zero, o que seria inviável, pois faria a extensão tender para infinito.

Embora os "monstros matemáticos" existissem há muito tempo, ainda ninguém lhes tinha atribuído um nome. Foi então que em 1978, Benoit Mandelbrot, ao preparar a sua primeira obra sobre os ditos "monstros", sentiu necessidade de lhes atribuir um nome, ficando então conhecido como "o pai dos fractais". Buscando uma definição para esta nova geometria podemos citar Oliveira: ,

A geometria fractal permite a representação de certos elementos naturais que possuem características irregulares. Com a geometria fractal torna-se possível a criação de modelos mais próximos da realidade. A geometria fractal fornece algoritmos para construção de formas idênticas às naturais e também ferramentas para o estudo das mesmas. Essa geometria vem se consolidando nos últimos anos com o desenvolvimento da tecnologia computacional e com auxílio de novas teorias nas áreas da física, biologia, astronomia e matemática. [6]

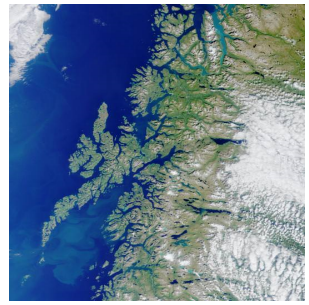

(a) Costa da Noruega

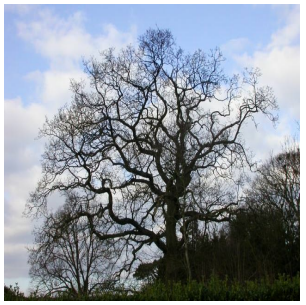

(b) Carvalho da África

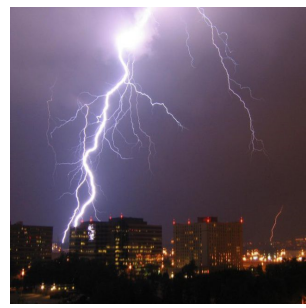

(c) Relâmpago

Figura 1: Fractais na natureza.

(Fonte: <http://cftc.cii.fc.ul.pt/PRISMA/capitulos/capitulo2/modulo4/topico6.php>.)

A definição mais simples é que fractais são objetos gerados pela repetição de um mesmo processo (iteração/recursão), apresentando autossimilaridade, dimensão fractal e complexidade infinita.

Os fractais vêm sendo aplicados nas mais diversas áreas das ciências e tecnologias. No desenvolvimento de tecnologias podemos citar a utilização de antenas fractais empregadas na telefonia celular, na transmissão wireless, na TV digital (HDTV), entre outras, conforme Figuras (2) (a) e (2) (b). Na agricultura, a análise de solos, nebulosidade da área, movimentos dos rios e estrutura de vários cristais podem ser modelados por fractais.

Na computação gráfica, a geometria fractal auxilia na criação de cenários naturais, como rios, conjuntos montanhosos e plantas, conforme Figuras (3) (a) e (3) (b). Tal teoria é útil também na 


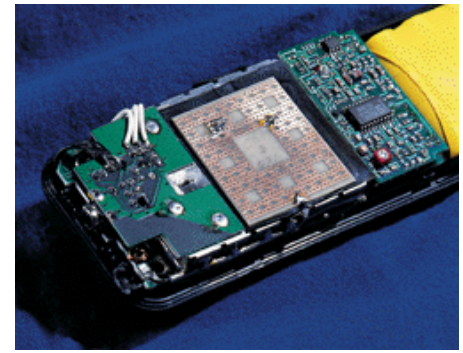

(a) Telefone celular

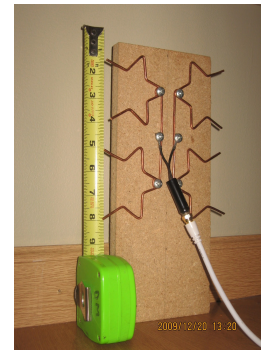

(b) Antena caseira

Figura 2: Telefone celular e antena caseira.

(Fontes:<http://cftc.cii.fc.ul.pt/PRISMA/capitulos/capitulo2/modulo4/topico9.php > e <https://www.pinterest.com/charley0810/diy-tv-antenna/)>.)

geração de efeitos especiais, como explosões e lavas de vulcões. Podemos dizer que a geometria fractal revolucionou a computação gráfica e, por outro lado, o advento do recurso computacional possibilitou o aprofundamento nos estudos dos fractais. Há aplicações também na economia, no

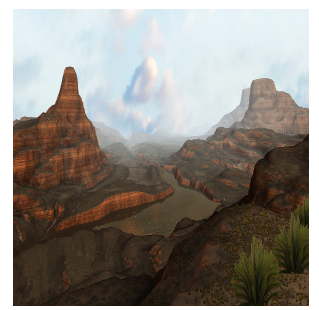

(a) Grand Canyon

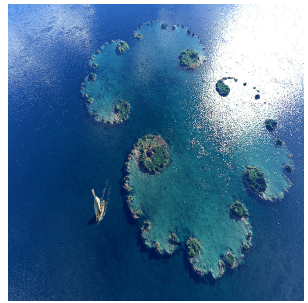

(b) Julia Island

Figura 3: Imagens geradas por Computação Gráfica.

(Fontes: <https://commons.wikimedia.org/wiki/File:Julia_island2.jpg> e <https://commons.wikimedia.org/wiki/File:Julia_island2.jpg)>.)

estudo da variação de preços das ações nas bolsas de valores. Na Astronomia, para a previsão de trajetórias futuras dos planetas. Em Mineralogia, para a medição da densidade dos minerais, evolução dos terrenos, descontinuidade das rochas. Na Geografia, a geometria fractal pode ser usada para a medição do comprimento da costa continental e na indústria, para a detecção automática de falhas têxteis.

Nas ciências médicas e biológicas, encontramos diversas estruturas que podem ser modeladas através da geometria fractal. Como exemplo, podemos citar as ramificações pulmonares, veias e artérias, cujos padrões podem ser bem representados por fractais, conforme as Figuras (4) (a) e (4) (b). Um ritmo cardíaco, apesar de aparentemente constante, tem variações aleatórias, porém, identificam-se padrões fractais nessas variações em diferentes escalas. Na Biologia, algumas plantas e micro-organismos apresentam estrutura fractal. É o caso, por exemplo da samambaia. 


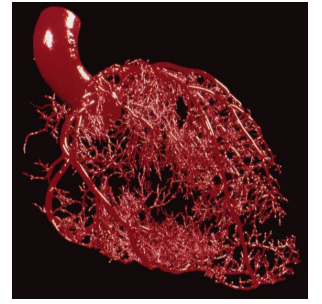

(a) Sistema arterial do coração

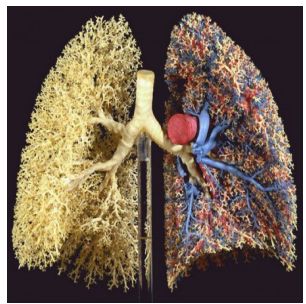

(b) Pulmão

Figura 4: Fractais no corpo humano.

(Fonte: <parquedaciencia.blogspot.com.br/2011_09_01_archive.html>.)

A análise de imagens no diagnóstico precoce de câncer pode ser feita através de modelagem utilizando-se os fractais, conforme Figuras (5) (a), (5) (b) e (5) (c).

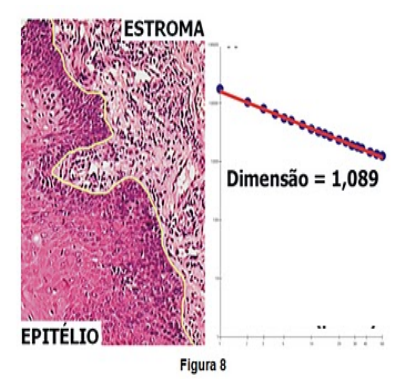

(a) Boca saudável

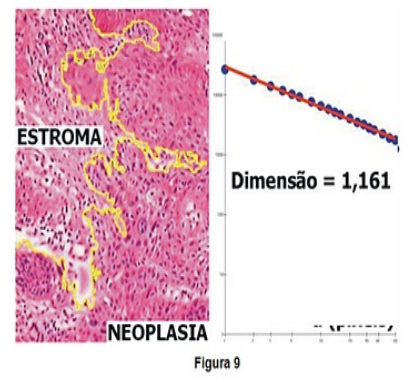

(b) Boca com neoplasia

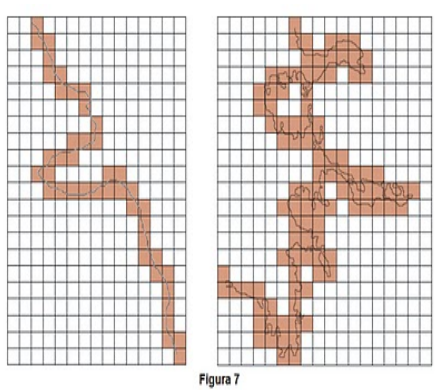

(c) Dimensão das caixas

Figura 5: Diagnóstico de câncer bucal usando dimensão fractal.

(Fonte: <parquedaciencia.blogspot.com.br/2011_09_01_archive.html>.)

\section{Atividades didáticas propostas}

A geometria fractal possui um vasto campo de aplicação dos conceitos matemáticos em suas diversas áreas, tais como álgebra, cálculo, geometria plana e espacial, progressões e logaritmo. Conforme será visto a seguir, é possível adequarmos alguns dos conceitos presentes na geometria fractal aos conteúdos curriculares.

As atividades descritas abaixo foram realizadas durante o Programa de Iniciação Científica da Escola SESC de Ensino Médio. No ano de 2013, o projeto foi desenvolvido com um grupo de três alunos, sendo dois do segundo e um do primeiro ano do ensino médio no período de Maio a Outubro, com culminância na semana de 28 de outubro a 1 de novembro de 2013, período em que ocorre a semana da Escola aberta, onde a instituição apresenta os trabalhos realizados pelos seus alunos no decorrer do ano para toda a comunidade escolar e instituições convidadas. 


\subsection{Construção do triângulo de Sierpinski}

A atividade apresentada a seguir foi adaptada a partir do material disponível no endereço <http: //fractalfoundation.org/resources/fractivities/sierpinski-triangle $>$ e acessado em 10 de abril de 2013. O objetivo desta atividade é ilustrar um dos princípios fundamentais dos fractais, a repetição de um mesmo padrão em diferentes escalas, e como essa forma complexa pode ser gerada por simples repetições.

- Material necessário: Papel A4, lápis, lápis de cor, régua, compasso e tesoura.

- As COMPetênCias trabalhadas nesta Atividade foram:

Semelhança de figuras;

Progressão Geométrica (PG);

Comprimento, Perímetro e Área do Triângulo de Sierpinski;

Conceito de Limite;

Limite de uma PG.

- Tempo Estimado - 6 aulas de 50 minutos

2 aulas de 50 minutos para a teoria de Progressão Geométrica e Soma da PG (finita e infinita);

2 aulas de 50 minutos para construção de cada triângulo e montagem do tapete;

2 aulas de 50 minutos para análise e conclusões à respeito do comportamento do perímetro e da área do triângulo de Sierpinski.

- Construção

A atividade consiste em desenhar usando régua e compasso um triângulo equilátero e marcar em cada lado seu ponto médio, ligando-os, com isso formando quatro novos triângulos. Nos 3 triângulos virados para cima, repetir o procedimento do ponto médio e assim sucessivamente, colorindo todos os triângulos invertidos. Após terem realizado a tarefa, monta-se um triângulo maior, usando-se os desenhos feitos por eles.

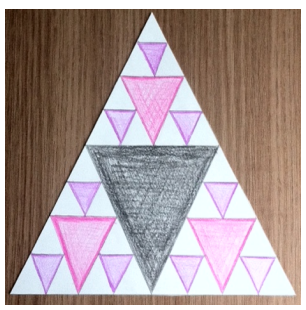

(a) Triângulo individual

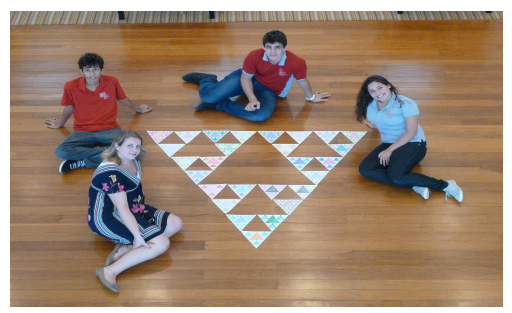

(b) Tapete de Sierpinski

Figura 6: Construção do Triângulo de Sierpinski até a $3^{\underline{a}}$ iteração pelos alunos da ESEM. 
- AnÁlise Observando a Figura (6) (a), começamos a analisar o comportamento da área e do perímetro do triângulo de Sierpinski. Partimos de um triângulo equilátero (figura inicial), depois removemos o triângulo equilátero definido pelos pontos médios dos lados e assim sucessivamente. O triângulo de Sierpinski é obtido como limite desse processo recursivo que está descrito, geometricamente, na Figura (7). Na $1^{1}$ iteração da construção, determinamos os pontos médios de
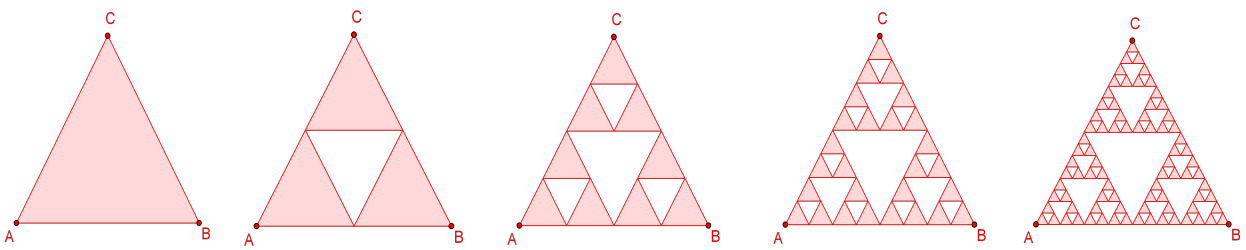

Figura 7: Iterações no triângulo de Sierpinski até a $4^{\underline{a}}$ iteração.

cada um dos lados do triângulo, unimos esses pontos médios (2 a 2) por segmentos e consideramos os 3 segmentos resultantes, retirando o triângulo central. Obtemos, portanto, a segunda figura do processo de construção. Repetindo indefinidamente o processo, obtemos o triângulo de Sierpinski no limite deste processo recursivo.

Observando a Figura (7), podemos verificar, que a cada iteração, a área do triângulo de Sierpinski, é igual à área do triângulo anterior multiplicada pelo fator $\frac{3}{4}$ e que o seu perímetro é igual ao perímetro do triângulo anterior multiplicado pelo fator $\frac{3}{2}=\left(3 \cdot \frac{1}{2}\right)$. Analisemos este fato através da tabela (8):

\begin{tabular}{cccc}
\hline Figura & Área & Perímetro & Área Esburacada \\
\hline Fig. Inicial & $A$ & $P$ & 0 \\
$1^{\mathrm{a}}$ iteração & $A_{1}=\frac{3}{4} \cdot A$ & $P_{1}=\frac{3}{2} \cdot P$ & $B_{1}=\frac{1}{4} \cdot A$ \\
$2^{\mathrm{a}}$ iteração & $A_{2}=\frac{3}{4} \cdot A_{1}=\left(\frac{3}{4}\right)^{2} \cdot A$ & $P_{2}=\frac{3}{2} \cdot P_{1}=\left(\frac{3}{2}\right)^{2} \cdot P$ & $B_{2}=\frac{1}{4} \cdot\left[A+\frac{3}{4} \cdot A\right]$ \\
$3^{\text {a }}$ interação & $A_{3}=\frac{3}{4} \cdot A_{2}=\left(\frac{3}{4}\right)^{3} \cdot A$ & $P_{3}=\frac{3}{2} \cdot P_{2}=\left(\frac{3}{2}\right)^{3} \cdot P$ & $B_{3}=\frac{1}{4} \cdot A \cdot\left[1+\frac{3}{4}+\frac{9}{16}\right]$ \\
$\vdots$ & $\vdots$ & $\vdots$ & $\vdots$ \\
$n^{\mathrm{a}}$ iteração & $A_{n}=\frac{3}{4} \cdot A_{n-1}=\left(\frac{3}{4}\right)^{n} \cdot A$ & $P_{n}=\frac{3}{2} \cdot P_{n-1}=\left(\frac{3}{2}\right)^{n} \cdot P$ & $B_{n}=\frac{1}{4} \cdot A \cdot\left[1+\frac{3}{4}+\cdots+\left(\frac{3}{4}\right)^{n-1}\right]$ \\
\hline
\end{tabular}

Figura 8: Área, Perímetro e Área Esburacada do triângulo de Sierpinski.

Analisando, a área do triângulo, temos uma $\mathrm{PG}$ de razão $\frac{3}{4}(|q|<1)$ e $1^{\circ}$ - termo positivo (pois $A$ é a área da figura inicial). É evidente que esta sucessão é monótona decrescente e a medida que o número de iterações se aproxima do infinito, ou seja, quando $n$ tende para infinito, $A_{n}$ tende a zero, isto é,

$$
\lim _{n \rightarrow \infty} A_{n}=0 .
$$

Isto significa que a área do triângulo de Sierpinski é igual a zero. Outra maneira de verificarmos este fato é analisarmos a área "esburacada", dada por:

$$
B_{n}=\frac{1}{4} \cdot A \cdot\left[1+\frac{3}{4}+\cdots+\left(\frac{3}{4}\right)^{n-1}\right]=\frac{1}{4} \cdot A \cdot S_{n}
$$


em que $S_{n}$ é a soma dos $n$ primeiros termos de uma PG, cujo primeiro termo é $a_{1}=1$ e a razão é $q=\frac{3}{4}$. Sabemos que para uma PG de razão $|q|<1$, a sequencia de números reais é convergente e seu limite é:

$$
\lim _{n \rightarrow \infty} S_{n}=\frac{a_{1}}{1-q}=\frac{1}{1-\frac{3}{4}}=\frac{1}{\frac{1}{4}}=4 .
$$

Portanto, a área dos buracos do triângulo, que denotaremos por $B_{\text {Sierpinski }}$, é tal que:

$$
B_{\text {Sierpinski }}=\lim _{n \rightarrow \infty} B_{n}=\lim _{n \rightarrow \infty}\left(\frac{1}{4} \cdot A \cdot S_{n}\right)=\frac{1}{4} \cdot A \cdot 4=A \text {. }
$$

Assim, quando $n \rightarrow+\infty, B_{\text {Sierpinski }} \rightarrow A$. Sabemos que $A=B_{n}+A_{n}$, ou seja, $B_{n}=A-A_{n}$. Como $B_{n} \rightarrow A$ então $A_{n} \rightarrow 0$. Logo, a área do triângulo de Sierpinski é igual a zero.

Analisando o perímetro do triângulo de Sierpinski temos uma PG de razão $q=\frac{3}{2}$ (maior que 1 ) e $1^{\text {o }}$ termo exatamente o perímetro da figura inicial (positivo). Assim, quando $n \rightarrow+\infty$, $P_{n} \rightarrow+\infty$. Isto significa, que o perímetro do triângulo é infinito.

\subsection{Construção da curva de Koch e da Ilha de Koch}

A atividade apresentada a seguir foi adaptada a partir do material disponível no seguinte endereço <http://fractalfoundation.org/resources/fractivities/koch-curve> e acessado em 10 de abril de 2013. Aqui destaca-se a importância de chamar atenção para o aluno do processo de repetição presente na construção. É possível associar esse fractal a linhas costeiras dos continentes, vendo aplicação direta da geometria fractal com a natureza.

- Material NeCessário: lápis, régua, tesoura e canetinha ou lápis de cor para colorir (opcional) e papel isométrico A3 que pode ser obtido no sítio <http://www.worksheetworks.com/ miscellanea/graph-paper/isometric-dots.html>.

- As COMPetênCias trabalhadAs nesta ATIVIDAdE FORAM:

Semelhança de figuras;

Progressão Geométrica;

Comprimento, Perímetro e Área da Ilha de Von Koch;

Conceito de Limite;

Limite de uma PG.

- Tempo Estimado - 4 aulas de 50 minutos

2 aulas de 50 minutos para construção de cada curva e montagem da ilha;

2 aulas de 50 minutos para análise e conclusões à respeito do comportamento dos lados, do perímetro e da área da ilha de Von Koch.

- Construção

A atividade consiste em desenhar no papel isométrico usando régua, um segmento de reta de medida múltipla de 3 para facilitar o desenvolvimento da atividade, usamos $27 \mathrm{~cm}$, dividimos esse segmento em 3 partes iguais, apagamos o segmento médio e fizemos um triângulo equilátero (sem 
a base), usando como medida um desses segmentos, com isso formamos quatro novos segmentos. Nos 4 novos segmentos, repetimos o procedimento descrito acima e assim sucessivamente. Feito isso, temos a curva de Von Koch. Unindo três desses desenhos, temos a Ilha de Von Koch. Os alunos optaram por colorir o desenho, para termos também um objeto de arte. Cortamos a ilha em formato hexagonal, pois a usaremos para auxiliar nos cálculos da área.

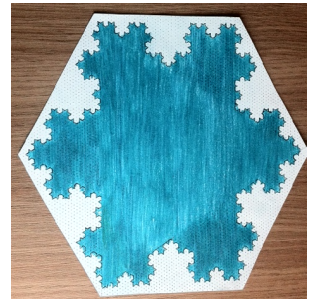

(a) Ilha de Koch individual

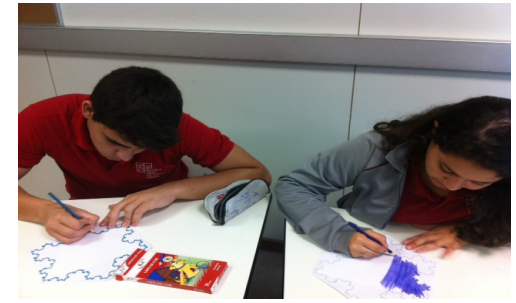

(b) Pintura da Ilha

Figura 9: Construção do Ilha de Von Koch até a $5^{\underline{a}}$ iteração.

\section{- AnÁlise}

Observando a Figura (8) (a), começamos a analisar o comportamento da área e do perímetro da ilha de Koch. Iniciamos o processo com um triângulo equilátero, na primeira iteração da construção dividimos cada lado do triângulo em três partes iguais e construímos sobre cada um dos segmentos do meio um novo triângulo equilátero, sem a base, tal como podemos observar na Figura (10). Obtivemos, portanto, a segunda figura do processo de construção (conhecida como "Estrela de Davi") com 12 lados. Repetimos o mesmo processo para cada um dos 12 segmentos obtidos na figura anterior. Repetindo indefinidamente o processo, obtemos, no limite deste processo recursivo, a curva de Koch.

Ao vermos a representação geométrica deste fractal, conforme Figura (10), podemos observar facilmente que possui uma forma regular fechada cuja fronteira é composta por infinitos lados cada vez menores. Supondo que cada lado do triângulo inicial mede uma unidade, observemos que para cada nova transformação que se faz, a quantidade de lados anterior é multiplicada por 4 e o comprimento dos lados de cada nova figura são 3 vezes menores que os da figura anterior. Analisemos este fato através da tabela 1, considerando o comprimento do lado do triângulo
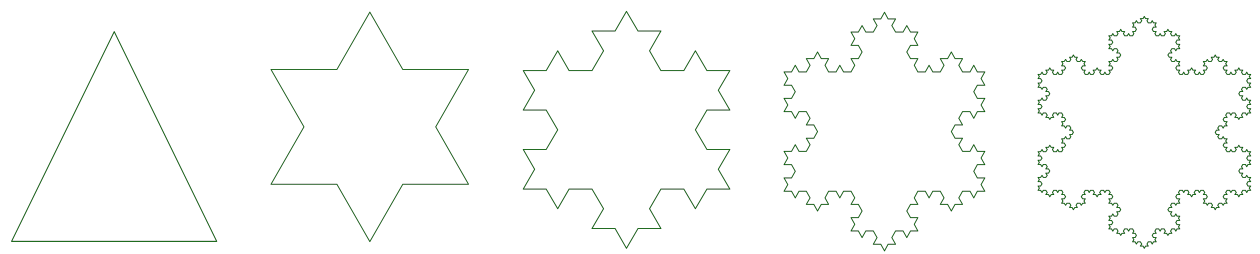

Figura 10: Iterações na ilha de Von Koch até a $4^{\underline{a}}$ iteração.

inicial igual a 1 unidade.

O número de lados de cada figura em função do número de iterações é dado por uma PG 


\begin{tabular}{ccc}
\hline Figura & Número de lados $(\mathrm{L})$ & Comprimento dos lados $(\mathrm{M})$ \\
\hline Fig. Inicial & $3 \cdot 1=3=3 \cdot 4^{0}$ & $M_{0}=1=3^{0}$ \\
$1^{\underline{a}}$ iteração & $3 \cdot 4=12=3 \cdot 4^{1}$ & $M_{1}=\frac{1}{3} \cdot M_{0}=3^{-1}$ \\
$2^{\underline{a}}$ iteração & $12 \cdot 4=48=3 \cdot 4^{2}$ & $M_{2}=\frac{1}{3} \cdot M_{1}=3^{-2}$ \\
$3^{\underline{a}}$ iteração & $48 \cdot 4=192=3 \cdot 4^{3}$ & $M_{3}=\frac{1}{3} \cdot M_{2}=3^{-3}$ \\
$4^{\underline{a}}$ iteração & $192 \cdot 4=768=3 \cdot 4^{4}$ & $M_{4}=\frac{1}{3} \cdot M_{3}=3^{-4}$ \\
$n^{\underline{a}}$ iteração & $3 \cdot 4^{n}$ & $3^{-n}$ \\
\hline
\end{tabular}

Tabela 1: Quantidade e Comprimento dos lados da Ilha de Von Koch.

em que o primeiro termo é $L_{0}=3$ e a razão é $q=4$. Tal progressão pode ser definida por recorrência ou através de um termo geral, conforme dado abaixo.

$$
L_{n}=\left\{\begin{array}{l}
L_{n}=3 \text { se } n=0 \\
L_{n}=4 \cdot L_{n-1} \text { se } n>1
\end{array} \quad \text { ou } \quad L_{n}=3 \cdot 4^{n}\right.
$$

É evidente que esta sucessão é monótona crescente e que, a medida que o número de iterações aproxima-se do infinito, ou seja, quando $n \rightarrow+\infty$, a sucessão $L_{n} \rightarrow \infty$, isto é,

$$
\lim _{n \rightarrow \infty} L_{n}=+\infty
$$

Isto significa que a curva vai ter um número infinito de lados. O comprimento dos lados de cada figura em função do número de iterações, é dado também por uma $\mathrm{PG}$, onde $M_{0}=1$ e razão $q=\frac{1}{3} \quad(|q|<1)$, que pode ser definida por recorrência ou através de um termo geral

$$
M_{n}=\left\{\begin{array}{l}
M_{n}=1 \text { se } n=0 \\
M_{n}=\frac{1}{3} \cdot M_{n-1} \text { se } n>1
\end{array} \quad \text { ou } \quad M_{n}=\left(\frac{1}{3}\right)^{n}=3^{-n} .\right.
$$

É evidente que esta sucessão é monótona decrescente e limitada por zero e, à medida que o número de iterações se aproxima do infinito, ou seja, quando $n \rightarrow \infty$, a sucessão $M_{n} \rightarrow 0$. Isto é, $\lim _{n \rightarrow \infty} M_{n}=0$. Mostrando que o comprimento de cada lado da curva tende para zero. Seja

$$
P_{n}=M_{n} \cdot L_{n}=\left(3 \cdot 4^{n}\right) \cdot 3^{-n}=3 \cdot\left(\frac{4}{3}\right)^{n}
$$

o perímetro obtido na $n$-ésima iteração. Observa-se que $P_{n}$ é uma $\mathrm{PG}$ de razão $\frac{4}{3}$ (maior que 1) e $P_{0}=3$ ( $1^{\text {o }}$ termo, exatamente, o perímetro do triângulo inicial) e $P_{1}=4$ ( $P_{0}$ e $P_{1}$ ambos positivos). Assim, $P_{n} \rightarrow+\infty$ quando $n \rightarrow+\infty$. Isto significa que o perímetro da ilha de Von Koch é infinito.

Consideremos, para facilitar os cálculos, que A é a área do triângulo equilátero obtido na primeira iteração, então $A_{0}=A$. Comecemos por estimar a área da curva de Koch traçando um hexágono envolvendo a "Estrela de Davi" (iteração 1), ao continuarmos a construção, constatamos que a figura da $2^{\underline{a}}$ iteração ainda está contida no hexágono, conforme Figura (11). Note que é fácil perceber que isso vai acontecer em todas as iterações. Podemos então concluir que a área da ilha de Von Koch é inferior à área do hexágono, que é igual ao dobro da área do triângulo 

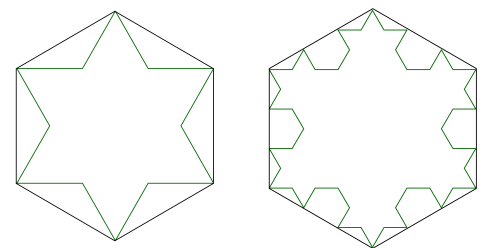

Figura 11: Ilha envolvida por um hexágono nas $1^{\underline{a}}$ e $2^{\underline{a}}$ iterações.

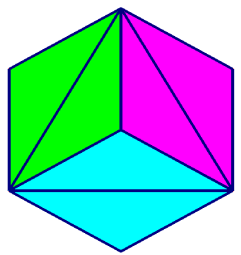

Figura 12: Área do hexágono em função de um triângulo equilátero.

inicial, conforme a Figura (12), portanto, $2 A$. Com isso, podemos perceber que a área da região delimitada pela ilha de Koch estará compreendida entre $A$ e $2 A$.

Sabemos que a área do polígono, em cada iteração, obtém-se adicionando a área do polígono da iteração anterior a área de um triângulo equilátero, cujo lado é $\frac{1}{3}$ do anterior, multiplicada tantas vezes quantas forem o número de lados do polígono anterior.

Nota-se que ao compararmos a construção iniciada com um triângulo equilátero de lado $l$ e área $A$ com a construção na qual iniciamos com um triângulo equilátero de lado $l^{\prime}=\frac{l}{3}$, obtemos a seguinte relação entre as áreas $A$ e $A^{\prime}$ :

$$
A^{\prime}=\frac{l^{\prime 2} \sqrt{3}}{2}=\frac{\sqrt{3}}{2}\left(\frac{l}{3}\right)^{2}=\frac{1}{9} \frac{l^{2} \sqrt{3}}{2}=\frac{1}{9} A .
$$

Fazendo um estudo sistemático:

Na figura inicial, temos o número de lados igual a $L_{0}=3 \cdot 4^{0}$ e área igual a $A_{0}=A$.

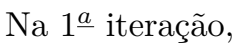

$$
L_{1}=3 \cdot 4^{1}, \quad A_{1}=A_{0}+L_{0} \cdot \frac{1}{9} \cdot A=A+3 \cdot\left(\frac{1}{9}\right)^{1} \cdot A
$$

$\mathrm{Na} 2^{a}$ iteração,

$$
\begin{aligned}
& L_{2}=3 \cdot 4^{2}, \quad A_{2}=A_{1}+L_{1} \cdot \frac{1}{9} \cdot\left(\frac{1}{9} \cdot A\right)=A_{1}+L_{1} \cdot\left(\frac{1}{9}\right)^{2} \cdot A \\
& A_{2}=A+3 \cdot\left(\frac{1}{9}\right) \cdot A+3 \cdot 4 \cdot\left(\frac{1}{9}\right)^{2} \cdot A=A+\frac{1}{3} \cdot A+\frac{1}{3} \cdot \frac{4}{9} \cdot A
\end{aligned}
$$


$\mathrm{Na} 3^{\underline{a}}$ iteração, temos:

$$
\begin{aligned}
& L_{3}=3 \cdot 4^{3}, \quad A_{3}=A_{2}+L_{2} \cdot\left(\frac{1}{9}\right)^{3} \cdot A=A+\frac{1}{3} \cdot A+\frac{1}{3} \cdot \frac{4}{9} \cdot A+3 \cdot 4^{2} \cdot\left(\frac{1}{9}\right)^{3} \cdot A \\
& A_{3}=A+\frac{1}{3} \cdot A+\frac{1}{3} \cdot \frac{4}{9} \cdot A+\frac{1}{3} \cdot\left(\frac{4}{9}\right)^{2} \cdot A=A+\frac{A}{3} \cdot\left[1+\frac{4}{9}+\left(\frac{4}{9}\right)^{2}\right]
\end{aligned}
$$

Portanto, na $n$-ésima iteração, a área da ilha de Von Koch tem a seguinte expressão:

$$
A_{n}=A+\frac{A}{3}\left[1+\frac{4}{9}+\left(\frac{4}{9}\right)^{2}+\ldots+\left(\frac{4}{9}\right)^{n-1}\right]=A+\frac{A}{3} S_{n}
$$

onde $S_{n}$ é a soma dos $n$ primeiros termos de uma PG, cujo $a_{1}=1$ e $q=\frac{4}{9}$.

Sabemos que para uma $\mathrm{PG}$ de razão $|q|<1$, a sequência de números reais é convergente e seu limite é:

$$
\lim _{n \rightarrow \infty} S_{n}=\frac{a_{1}}{1-q}=\frac{1}{1-\frac{4}{9}}=\frac{1}{\frac{5}{9}}=\frac{9}{5} .
$$

Portanto, a área da ilha de Von Koch, denotada por $A_{K o c h}$, será dada por:

$$
A_{K o c h}=\lim _{n \rightarrow \infty} A_{n}=\lim _{n \rightarrow \infty}\left(A+\frac{A}{3} \cdot S_{n}\right)=A+\frac{A}{3} \cdot \frac{9}{5}=A+\frac{3 A}{5}=\frac{8}{5} \cdot A=1,6 \cdot A,
$$

donde concluímos que, quando $n \rightarrow \infty, A_{\text {Koch }} \rightarrow 1,6 \cdot A$

Nota-se que, embora o perímetro seja infinito (ilimitado superiormente), a área tem um limite finito e bem definido igual a 1,6 vezes a área inicial.

\subsection{Fractais no $X$ aos}

Hoje em dia alguns programas para gerar fractais são tão eficientes que nos permitem "navegar" pelas imagens em movimento enquanto elas são produzidas. É como um mergulho pelos detalhes infinitos onde o usuário escolhe com o mouse o caminho que quer fazer enquanto a imagem é constantemente ampliada e os novos detalhes aparecem e se transformam continuamente. São descritas abaixo algumas atividades que foram desenvolvidas com os alunos da Escola SESC de Ensino Médio utilizando o software Xaos em sua versão 3.5 .

Inicialmente apresenta-se algumas observações sobre o software. Ele pode ser obtido gratuitamente em Português no seguinte endereço: <http://sourceforge.net/projects/xaossomething >.

1. Recentemente foi disponibilizada uma versão gratuita do Xaos para Iphone e Ipad;

2. A interface é simples e eficiente, conforme Figura (13);

3. Oferece movimento de navegação, você pode aumentar (zoom in) ou diminuir (zoom out) um fractal;

4. Inclui 24 fórmulas de fractais conhecidos, além de diversas variações possíveis em efeitos e aparência, mas você também pode usá-lo para gerar seus próprios fractais, conforme Figura (14);

5. Oferece efeitos como um gerador de Starfield, pode gravar, tem trilha de movimento, modo de pseudo $3 \mathrm{D}$, e até mesmo um display de texto; 


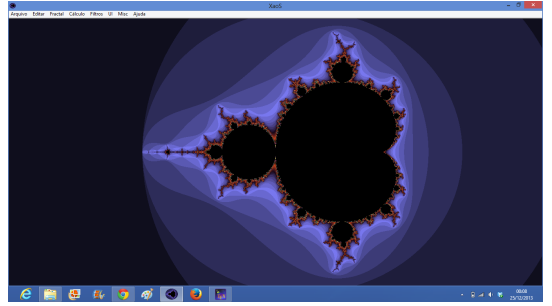

Figura 13: Tela inicial do Xaos 3.5.

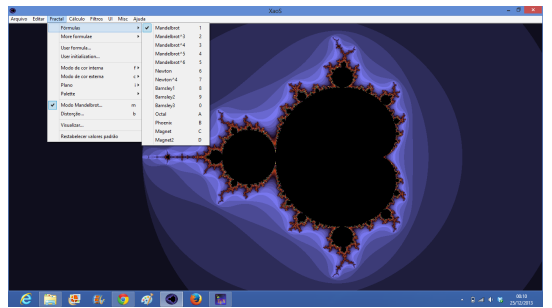

Figura 14: Tela com alguns exemplos de fractais do Xaos 3.5.

6. Pode controlar o zoom com o mouse, tanto para ampliar como para diminuir, conforme Figura (15);
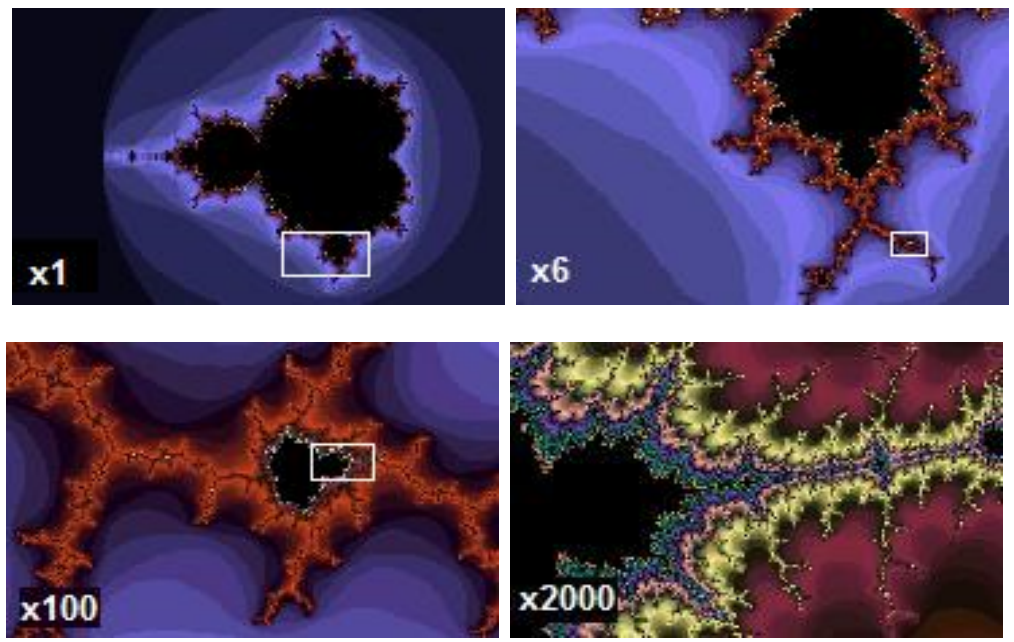

Figura 15: Zoom do Conjunto de Mandelbrot no Xaos.

(Fonte: <http://en.wikipedia.org/wiki/Fractal>)

7. Há um arquivo de ajuda que oferece tutoriais animados explicando o que são fractais, como o programa funciona, e suas muitas opções de configuração. Estes incluem menus para a escolha de fractais, definindo seus cálculos matemáticos, aplicação de filtros e controlar o movimento do programa;

8. A maneira mais simples para se familiarizar com Xaos, é clicar em uma parte do fractal e começar a dar 
zoom.

Os alunos puderam interagir no Xaos, criando os mesmos fractais que já haviam criado em sala de forma manual. O que os deixou encantados foi a beleza, o colorido, o movimento e a possível manipulação com um número elevado de iterações. Conforme as Figuras (15), (16) e (17).

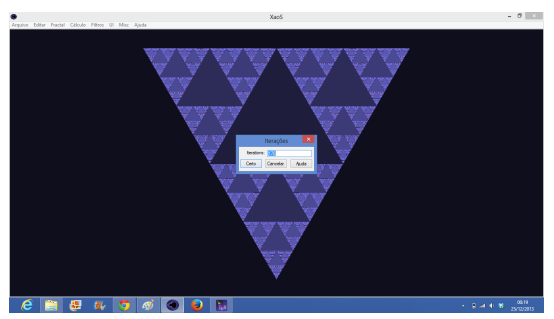

Figura 16: Tela com o triângulo de Sierpinski com 170 iterações do Xaos 3.5.

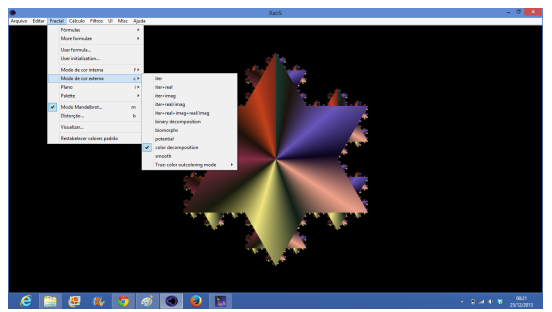

Figura 17: Tela com a Ilha de Koch colorida do Xaos 3.5.

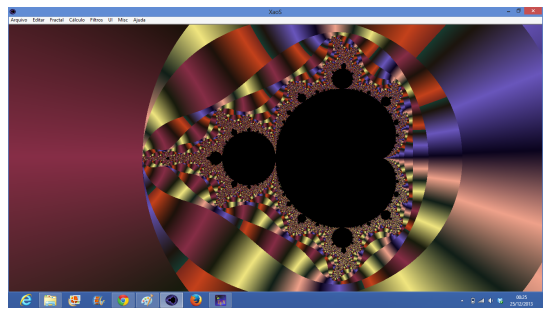

Figura 18: Tela com o conjunto de Mandelbrot do Xaos 3.5.

\section{Considerações finais}

As atividades foram aplicadas a um grupo de três alunos da Escola SESC de Ensino Médio, como Projeto de Iniciação Científica, que ao início do ano letivo optaram por fazer parte desta pesquisa, tendo como característica comum o interesse por temas ligados à Matemática. Isto, de certa forma, facilitou as aplicações propostas. O grupo já possuía um conceito prévio da Geometria Euclidiana e introduzimos aulas teóricas com conceitos intuitivos de limites, progressões e leis de formação, então durante a realização das atividades propostas, o grupo foi conduzido a chegar ao conceito mais simples de fractal e as suas características básicas. 
Com o estudo sistemático, por exemplo, da "Ilha de Koch" que apresenta perímetro infinito e área finita, a curiosidade dos alunos foi surgindo naturalmente. Com isso percebemos que, a construção e estudo do comportamento deste fractal, pode ser uma boa forma de consolidar conhecimentos já adquiridos envolvendo fórmulas algébricas, áreas e perímetros, assim como o cálculo do número de segmentos. Além disso, através das atividades realizadas, os alunos não só conheceram imagens fractais como também puderam reforçar os conceitos apresentados: de iterações e de autossemelhança. Além, é claro, de como já citado acima, os conceitos de progressão geométrica e de limite.

A cada semana, o grupo estava mais entrosado e coeso com a aplicação e realização das atividades, sempre demonstrando interesse de aprender o que estava sendo mostrado pelo professor e ir além em suas pesquisas e indagações, tornando o trabalho gratificante. Conseguimos, através da construção dos fractais (manual ou eletronicamente), cativar o interesse dos alunos para a aplicação também dos conceitos teóricos e não apenas da prática. Com a finalização do prazo das pesquisas e desenvolvimento, os alunos prepararam a apresentação do trabalho para ser divulgado na semana da Escola Aberta, onde realizaram a apresentação do que haviam aprendido durante as semanas de realização do projeto. O grupo foi elogiado tanto pelos colegas quanto pela coordenação da escola pelo desenvolvimento do projeto.

Ressaltamos, que o grupo de alunos, apesar de pequeno, produziu resultados bastante proveitosos. Esperamos que este fato sirva de estímulo aos professores para que repitam a gratificante experiência.

\section{Referências}

[1] Barbosa, R. M. Descobrindo a Geometria Fractal para a sala de aula. Terceira edição. Autêntica, Belo Horizonte, 2002.

[2] Batanete, A. et al. Natureza: Caos ou Ordem?. Universidade de Coimbra, Faculdade de Ciências e Tecnologia - Departamento de Matemática, Coimbra, 2004-2005.

[3] Bemfica, A.; Alves, C. Fractais: Progressão e série geométrica? Uma Metodologia de Ensino. Faculdade Cenecista de Osório (FACOS), Rio Grande do Sul, 2010.2.

[4] Mandelbrot, B. B. The Fractal Geometry of Nature. W. H. Freeman, San Francisco, 1982.

[5] Nunes, R. S. R. Geometria Fractal e Aplicações. Departamento de Matemática Pura.Faculdade de Ciências da Universidade do Porto, Porto, 2006.

[6] Oliveira, D. A Geometria Fractal no Ensino Fundamental e Médio. Faculdade de Filosofia, Ciências e letras de Presidente Venceslau (FAFIPREVE). São Paulo, 2008.

Ivana Côrtes

Escola SESC de Ensino Médio

<ivana.rccortes@gmail.com>

Gladson Antunes

UNIRIO

<gladson.antunes@uniriotec.br>

Recebido: 2014

Publicado: 2014 